\title{
Kesirli telegraf k1smi diferansiyel denklemin varyasyonel iterasyon metoduyla çözümü
}

\author{
Mahmut MODANLI ${ }^{1, *}$, Ayşe AKSOY ${ }^{2}$ \\ Harran Üniversitesi Fen-Edb. Fak. Matematik Böl., Osmanbey kampüsü, Şanlıurfa. \\ Harran Üniversitesi Fen Bilimleri Enstitüsü, Osmanbey kampüsü, Şanlıurfa. \\ Geliş Tarihi (Received Date): 09.03.2021 \\ Kabul Tarihi (Accepted Date): 17.09.2021
}

$\ddot{\mathbf{O} z}$

Bu çalışmada, Caputo türeviyle tanımlı kesirli mertebeden telegraf kısmi diferansiyel denkleminin başlangıç-sınır değer koşullarına bağlı yaklaşık çözümü incelendi. Bu denklem için varyasyonel iterasyon metodunun çözüm prosedürü sunuldu. Bu metot için Lagrange parametresi belirlenip doğrulama fonksiyoneli oluşturuldu. Kesirli mertebeden telegraf kismi diferansiyel denklemin örnek bir probleminin verilen başlangıç değerleri kullanılarak varyasyonel iterasyon metodu ile nümerik çözümleri elde edildi.

Anahtar kelimeler: Kesirli telegraf kismi diferansiyel denklemi, varyasyonel iterasyon metodu, Langrange parametresi, yaklaşık çözüm.

\section{On the numerical solution of the fractional telegraph partial differential equation with variational iteration method}

\begin{abstract}
In this work, the fractional order telegraph partial differential equation defined by Caputo derivative depend on initial-boundry value conditions is investigated for approximate solutions. The solution procedure of the variational iteration method is presented for this fractional order telegraph partial differential equation. Lagrange parameter is determined and correction functional is formed. The numerical solutions of an example problem for the fractional order telegraph partial differential equation are obtained by variational iteration method by using the given initial values.
\end{abstract}

\footnotetext{
*Mahmut MODANLI, mmodanli@harran.edu.tr, http://orcid.org/0000-0002-7743-3512 Ayşe AKSOY, aysee-fzl@hotmail.com, http://orcid.org/0000-0001-7179-0124
} 
Keywords: Fractional telegraph partial differential equations, variational iteration method, Langrange parameter, approximate solution

\section{Giriş}

Kesirli analiz konusu son yıllarda büyük bir önem kazanmış, bu alanda çalışan birçok insanın ilgisini çekmiş, ayrıca fen ve mühendisliğin çeşitli alanlarında kullanılmıştır. Tam sayı olmayan mertebeye sahip türev ve integralle ilgili en ünlü olan RiemannLiouville ve Caputo tanımlarıdır. Caputo, kesirli mertebeden diferansiyel denklemleri çözerken tamsayı mertebeden başlangıç şartlarını kullanmak için Riemann-Liouville kesirli türev tanımını yeniden formüle etmiştir [1]. Kesirli operatörler üzerinde büyük işleve sahip olan gama ve beta fonksiyonlarının tanımı şu şekildedir.

\subsection{Temel Kavramlar}

\section{Tanım 1.1.1.}

$z \in \mathbb{C}$ ve $\operatorname{Re}(z)>0$ olsun. Gama fonksiyonu,

$\Gamma(z)=\int_{0}^{\infty} t^{z-1} e^{-t} d t$

integrali ile tanımlanır. $\Gamma(z)$ ile gösterilir.

Tanım 1.1.2.

$\operatorname{Re}(m)>0$ ve $\operatorname{Re}(n)>0$ olsun. Beta fonksiyonu,

$B(m, n)=\int_{0}^{1} t^{m-1}(1-t)^{n-1} d t$

olarak tanımlanır [2-3].

Tanım 1.1.3.

Riemann-Liouville integralinin $n-1<\alpha \leq n$ olmak üzere $\alpha$. mertebeden tanımı

$I^{\alpha}(f(x))=\frac{1}{\Gamma(\alpha)} \int_{0}^{x} \frac{f(s) d s}{(x-s)^{1-\alpha}}$

olarak verilmiştir [1].

Tanım 1.1.4.

$p$. mertebeden Riemann-Liouville kesirli türev tanımı ise $m<p \leq m+1$ olmak üzere,

$D_{t}^{p} f(t)=\left(\frac{d}{d t}\right)^{m+1} \frac{1}{\Gamma(m-p+1)} \int_{a}^{t}(t-\tau)^{m-p} f(\tau) d \tau$

olarak tanımlanmıştır [1].

Tanım 1.1.5.

Zamana bağlı $\alpha$ 1ncı mertebeden $D_{t}^{\alpha} u(t, x)$ Caputo kesirli türevi $n-1<\alpha \leq n$ için 
$D_{t}^{\alpha} u(t, x)=\frac{\partial^{\alpha} u(t, x)}{\partial t^{\alpha}}=\frac{1}{\Gamma(n-\alpha)} \int_{0}^{t} \frac{1}{(t-p)^{\alpha-n+1}} \frac{\partial^{n} u(p, x)}{\partial p^{n}} d p$

ve $\alpha=n \in \mathbb{N}$ için

$D_{t}^{\alpha} u(t, x)=\frac{\partial^{\alpha} u(t, x)}{\partial t^{\alpha}}=\frac{\partial^{n} u(t, x)}{\partial t^{n}}$

olarak tanımlanır [4].

Riemann-Liouville türevi yerine Caputo türevinin tercih edilmesinin bir sebebi Caputo türevinde sabitin türevi sıfır olurken, Riemann-Liouville türevinde sabitin türevi sıfirdan farklıdır. Kesirli mertebeden türevler fikri ilk olarak yedinci yüzyılda büyük matematikçi Newton ve Leibnitz tarafından tanımlanmış ve akışkanlar mekaniği, sönümleme yasaları, elektrik ağları, sinyal işleme, difüzyon-reaksiyon süreci, gevşeme süreçleri, elektrokimya, matematiksel biyoloji, fizik, mühendislik ve diğer bilim dallarındaki çeşitli önemli fenomenlerde ortaya çıkan lineer olmayan karmaşık sistemlerdeki sayısız uygulamaları nedeniyle büyük bir ilgi görmüştür. Kesirli türevler tam sayı mertebeli türevlere kıyasla gerçek hayat problemlerinin daha hassas modellerini sunar. $\mathrm{Bu}$ bağlamda depremin lineer olmayan salınımı kesirli türevlerle modellenebilir [5] kesirli türevli akışkan-dinamik trafik model [6], sürekli trafik akımı varsayımından ve gözenekli ortamda sızıntı akışı için kesirli lineer olmayan karmaşık modelden kaynaklanan eksikliği ortadan kaldırabilir [7]. Kesirli denklemler bu ve bunun gibi daha pek çok uygulama alanlarında kullanılabilir. Zamana bağlı kesirli diferansiyel denklemlerin birçok değişik tipi vardır. Kesirli Klein-Gordon denklemleri dalga etkileşimini tanımlamak için kuantum alan teorisinde, kuantum mekaniğinde ve yoğun madde fiziğinde kullanılır [8-9]. Kesirli Burger's denklemleri viskoelastik sıvıların kararsız akışlarını kanal (halka) tüpü ve hız alanı çözümleri aracılığıyla araştırmak için ortaya çıkmıştır [10]. Kesirli Black-Scholes Avrupa opsiyon fiyatlandırma denklemleri finansal pazar için en kayda değer matematik modellerinden biri olan bu problem çalışıldı [11]. Biyoloji, tıp, popülasyon ekolojisi, kontrol sistemleri, iklim modelleri [12] ve karmaşık ekonomik makro dinamikler [13] alanında ortaya çıkan orantılı gecikmeli zamana bağlı kesirli kısmi diferansiyel denklemlerin başlangıç değerli bağımsız sistemleridir [14]. Aslında kesirli mertebeden diferansiyel denklemlerin geniş bir sınıfının tam çözümünü hesaplamak çok zorlu bir iştir. Geçtiğimiz yıllarda bu tipteki diferansiyel denklemlerin kesirli modelinin yaklaşık çözümünü bulmak için güçlü tekniklerin farklı türleri tanıtılmıştı. Bunlardan bazıları genelleştirilmiş diferansiyel dönüşüm metodu [15], Adomian ayrıştırma metodu [16], homotopi analiz metodu [17], homotopi analiz dönüşüm metodu [18], Chebyshev pseudo spektral metodu [19], değiştirilmiş Laplace dönüşümü metodu [20], Sumudu dönüşümü metodu [11,21], grup analiz metodu [22], dinamik akış modeli [23] ve homotopi pertürbasyon dönüşümü metodudur [24-26]. [27] de çalışılan denklem Homotopi analiz metodunu kullanarak $1<\alpha \leq 2$ aralığı için çözüldü. Varyasyonel iterasyon metodu Çinli matematikçi He [7] tarafından geliştirilmiştir. He'nin ufuk açıcı çalışmasından sonra, Cantor kümeleri üzerinde difüzyon ve dalga denklemleri [28], Riccati diferansiyel denklemi [29], iki boyutlu Burger's denklemlerinin kesirli modeli [30], Fisher's denklemleri, lineer olmayan osilatörler, Evrim denklemleri, tekil problemler, gözenekli bir ortam boyunca kararsız akım, sızıntı akımı, bağımsız adi diferansiyel denklemler, Korteweg-de Vries denklemleri, parabolik denklemler, integral-diferansiyel denklemleri, kimyasal problemler, Boussinesq denklemleri, Schrödinger denklemleri, Helmholtz denklemleri, Sine-Gordon denklemleri ve zamana 
bağlı kesirli Fornberg-Whitham denklemleri [31] bunların fiziksel doğasıyla uyum içerisinde varyasyonel iterasyon metodunun önerileri uyarınca başarılı bir şekilde çözülmeye çalışılmıştır [32-33]. Sonlu fark şeması metodu kullanılarak üçüncü mertebeden kısmi diferansiyel denklemler ve telegraf denklemlerin yaklaşık çözümleri verildi [34-36]. Bunlarla beraber, Kesirli mertebeden kısmi diferansiyel denklemlerin yaklaşık çözümleri ile ilgili pek çok çalışma yapılmıştır [37-45].

Varyasyonel iterasyon metodundaki Lagrange çarpanının kullanılmasının integral operatörünün ardışık uygulamalarını azalttığı, doğruluğu çok yüksek bir seviyede korurken hesaplama işini somut bir seviyeye indirdiği ve dolayısıyla bu tekniğin açık bir şekilde ayrıştırma metodundan daha avantajlı olduğu vurgulanmıştır. Başlangıç ve sınır koşullarını gerektiren değişkenlerine ayırma metodundan farklı olarak varyasyonel iterasyon metodu, sadece başlangıç koşullarını kullanarak çözüm sağlar. Varyasyonel iterasyon metodu ayrıca küçük parametre tahmininden bağımsızdır ve bu yüzden geleneksel perturbasyon metoduyla karşılaştırıldığında uygulaması daha uygundur. Varyasyonel iterasyon metodunun hiçbir ayrıştırma, sınırlayıcı tahmin veya dönüşüm olmaksızın tüm lineer ve lineer olmayan denklemlere uygulanabildiğine ve yuvarlama hatalarından uzak olduğuna da değinmek gerekir.

Tamsayı olmayan dereceli RLC elektrik devrelerinin dinamiği, kesirli mertebeden bir telegraf diferansiyel denklem ile tanımlandı [46]. Bu denklemde alçak geçiren, yüksek geçiren filtre karakteristiklerinin ve bant geçiren filtreler üç farklı filtreler elde edildi. Telgraf diferansiyel denklemi, zamana ve mekana bağlı şönt parametreleri ve zamana bağlı seri parametreleri için enerji nakil hatlarının eksiksiz bir matematiksel modelidir [47].

\section{Varyasyonel iterasyon metodu}

$\mathrm{Bu}$ yöntemde genel bir Lagrange parametresi ile doğrulama fonksiyoneli oluşturulur. Başlangıç koşullarından yola çıkarak iterasyonlar sonucu yaklaşık çözüm, bazen tam çözüm veya tam çözüme oldukça yakın bir çözüm bulunur. Bu problemin varyasyonel iterasyon metodu ile yaklaşık çözümünün elde edilmesi bu çalışmayı diğer çalışmalardan farklı kılmaktadır. Aynı zamanda bu problem hem $t$ hem de $x$ e göre bir telegraf kısmi diferansiyel denklemdir. Varyasyonel iterasyon metodunda bilinen $\hat{\mathrm{u}}_{\mathrm{k}}=0$ ve $\delta \mathrm{u}_{\mathrm{n}}(0, \mathrm{x})=0$ ifadelerinden başka herhangi bir varsayım kullanılmamıştır. $\mathrm{Bu}$ çalışmada aşağıdaki başlangıç ve sınır değer şartları ile verilen kesirli mertebeden telegraf kısmi diferansiyel denklem için

$$
\begin{aligned}
& \frac{\partial^{2} u(t, x)}{\partial t^{2}}+\frac{\partial^{x} u(t x)}{\partial t^{x}}+u(t, x)=\frac{\partial^{2} u(t, x)}{\partial x^{2}}+\frac{\partial u(t, x)}{\partial x}+f(t, x), \\
& 0<x<L, 0<t<T, 0<a \leq 1, \\
& u(0, x)=\varphi_{1}(x), u_{t}(0, x)=\varphi_{2}(x), 0 \leq x \leq L, \\
& u(t, 0)=u(t, L)=0,0 \leq t \leq T
\end{aligned}
$$

varyasyonel iterasyon metodu geliştirilecek ve çözüm prosedürü oluşturulacaktır. Burada $u=u(t, x)$ bilinmeyen fonksiyon, $f(t, x)$ bilinen fonksiyondur, $T$ ve $L$ birer pozitif reel sayıdır.

[48] çalışmasında Lagrange parametresinin tayini, başlangıç tahmini, doğrulama fonksiyoneli ve çözüm prosedürünün oluşturulması üzerinde durmuştur. Buna göre, 


$$
\left\{\begin{array}{l}
u_{0}(t, x)=V_{0}(t, x)+t V_{1}(t, x) \\
u_{n+1}(t, x)=u_{n}(t, x) \\
+\int_{0}^{t}(\xi-t)\left[\frac{\partial^{2} u_{n}(\xi, x)}{\partial \xi^{2}}+\frac{\partial^{\alpha} u_{n}(\xi, x)}{\partial \xi^{\alpha}}+u_{n}(\xi, x)-\frac{\partial^{2} u_{n}(\xi, x)}{\partial x^{2}}-\frac{\partial u_{n}(\xi, x)}{\partial x}-f(\xi, x)\right] d \xi
\end{array}\right.
$$

şeklinde başlangıç tahmini ve doğrulama fonksiyoneli elde edilip varyasyonel iterasyon formülü oluşturulur. Öncelikle bu metotta geçen Lagrange parametresinin $\lambda$ nasıl tayin edilebileceğini irdeleyelim.

Lineer ve lineer olmayan operatörler içeren bu denklemde Lagrange çarpanının tayini için [32] makalesini inceleyelim.

$$
L u(t)+N u(t)=g(t), t>0
$$

şeklinde verilen denklemde $L=\frac{d^{m}}{d t^{m}}, m \in \mathbb{N}$ lineer operatör, $N$ lineer olmayan operator ve $g(t)$ bilinen bir analitik fonksiyondur. (2.3) denklemi için doğrulama fonksiyoneli varyasyonel iterasyon metodu kullanılarak

$$
u_{k+1}(t)=u_{k}(t)+\int_{0}^{t}\left[\lambda(\xi)\left(L u_{k}(\xi)+N \tilde{u}_{k}(\xi)-g(\xi)\right)\right] d \xi
$$

inşa edilebilir. Burada Lagrange çarpanı $\lambda(\xi)$ varyasyon teorisi vasıtasıyla en uygun şekilde tanımlanabilir. Burada $\mathrm{Nu}$ lineer olmayan terime kısıtlı varyasyon uygulayalım. $\tilde{\mathrm{u}}_{\mathrm{k}}=0$ olacak şekilde yapalım. Bu durumda Lagrange çarpanının en iyi değeri genellikle

$\lambda(\xi)=\frac{(-1)^{m}}{(m-1) !}(\xi-t)^{m-1}, m \geq 1$

olarak uygulanır. (2.1) de verilen kesirli mertebeden telegraf kısmi diferansiyel denkleminde $m=2$ olarak alındığı için (2.4) denkleminde ifade edilen Lagrange çarpanı

$$
\lambda(\xi)=(\xi-t)
$$

olarak bulunur. Bu bilgilendirme 1şı̆̆ında uygulamaya (2.2) denklemi üzerinde devam edelim. Basit bir düzenleme yapılırsa

$$
\begin{aligned}
u_{n+1}(t, x)= & u_{n}(t, x)+\int_{0}^{t} \lambda(\xi) \frac{\partial^{2} \mathrm{u}_{n}}{\partial \xi^{2}}(\xi, x) d \xi+\int_{0}^{t} \lambda(\xi) \frac{\partial^{\alpha} \hat{\mathrm{u}}_{n}}{\partial \xi^{\alpha}}(\xi, x) d \xi \\
& +\int_{0}^{t} \lambda(\xi) \hat{\mathrm{u}}_{n}(\xi, x) d \xi-\int_{0}^{t} \lambda(\xi) \frac{\partial^{2} \tilde{\mathrm{u}}_{n}}{\partial x^{2}}(\xi, x) d \xi-\int_{0}^{t} \lambda(\xi) \frac{\partial \hat{\mathrm{u}}_{n}}{\partial x}(\xi, x) d \xi \\
& -\int_{0}^{t} \lambda(\xi) f(\xi, x) d \xi
\end{aligned}
$$

elde edilir. $\delta u_{n}(0, x)=0$ olmak üzere eşitliğin varyansı alınırsa 


$$
\begin{aligned}
\delta u_{n+1}(t, x)= & \delta u_{n}(t, x)+\int_{0}^{t} \lambda(\xi) \delta\left(\frac{\partial^{2} \mathrm{u}_{n}}{\partial \xi^{2}}(\xi, x)\right) d \xi+\int_{0}^{t} \lambda(\xi) \delta\left(\frac{\partial^{\alpha} \mathrm{u}_{n}}{\partial \xi^{\alpha}}(\xi, x)\right) d \xi \\
& +\int_{0}^{t} \lambda(\xi) \delta\left(\tilde{\mathrm{u}}_{n}(\xi, x)\right) d \xi-\int_{0}^{t} \lambda(\xi) \delta\left(\frac{\partial^{2} \hat{\mathrm{u}}_{n}}{\partial x^{2}}(\xi, x)\right) d \xi \\
& -\int_{0}^{t} \lambda(\xi) \delta\left(\frac{\partial \hat{\mathrm{u}}_{n}}{\partial x}(\xi, x)\right) d \xi-\int_{0}^{t} \lambda(\xi) \delta(f(\xi, x)) d \xi
\end{aligned}
$$

olup buradan da

$$
\begin{aligned}
\delta u_{n+1}(t, x)= & \delta u_{n}(t, x)+\int_{0}^{t} \lambda(\xi)\left(\frac{\partial^{2}}{\partial \xi^{2}} \delta \mathrm{u}_{n}(\xi, x)\right) d \xi+\int_{0}^{t} \lambda(\xi)\left(\frac{\partial^{\alpha}}{\partial \xi^{\alpha}} \delta \hat{\mathrm{u}}_{n}(\xi, x)\right) d \xi \\
& +\int_{0}^{t} \lambda(\xi)\left(\delta \hat{\mathrm{u}}_{n}(\xi, x)\right) d \xi-\int_{0}^{t} \lambda(\xi)\left(\frac{\partial^{2}}{\partial x^{2}} \delta \hat{\mathrm{u}}_{n}(\xi, x)\right) d \xi \\
& -\int_{0}^{t} \lambda(\xi)\left(\frac{\partial}{\partial x} \delta \tilde{\mathrm{u}}_{n}(\xi, x)\right) d \xi-\int_{0}^{t} \lambda(\xi)(\delta f(\xi, x)) d \xi
\end{aligned}
$$

elde edilir. $\delta \tilde{u}_{n}=0$ ve $\delta \mathrm{f}=0$ ifadelerinin kullanılmasıyla

$\delta u_{n+1}(t, x)=\delta u_{n}(t, x)+\int_{0}^{t} \lambda(\xi)\left(\frac{\partial^{2}}{\partial \xi^{2}} \delta u_{n}(\xi, x)\right) d \xi$

bulunur. İki kez kısmi integrasyonun uygulaması ile

$\delta u_{n+1}(t, x)=\delta u_{n}(t, x)\left(1-\lambda^{\prime}(\xi)\right)+\left.\lambda(\xi) \delta u_{n}^{\prime}(\xi, x)\right|_{\xi=t}+\int_{0}^{t} \lambda^{n}(\xi) \delta \mathrm{u}_{n}(\xi, x) d \xi=0$

elde edilir. Buradan da

$$
\left\{\begin{array}{c}
1-\lambda^{\prime}(\xi)=\left.0\right|_{\xi=t^{\prime}} \\
\lambda(\xi)=\left.0\right|_{\xi=t^{\prime}} \\
\lambda^{n}(\xi)=\left.0\right|_{\xi=t}
\end{array}\right.
$$

olarak bulunur. Böylece Lagrange çarpanı

$\lambda=(\xi-t)$

olarak elde edilir. Böylece kesirli mertebeden telegraf kısmi diferansiyel denklemi için Lagrange çarpanı (2.2) denkleminde verilen doğrulama fonksiyonelinde yerine yazılırsa 


$$
\begin{aligned}
u_{n+1}(t, x)= & u_{n}(t, x) \\
& +\int_{0}^{t}(\xi-t)\left[\frac{\partial^{2} u_{n}(\xi, x)}{\partial \xi^{2}}+\frac{\partial^{\alpha} u_{n}(\xi, x)}{\partial \xi^{\alpha}}+u_{n}(\xi, x)-\frac{\partial^{2} u_{n}(\xi, x)}{\partial x^{2}}\right. \\
& \left.-\frac{\partial u_{n}(\xi, x)}{\partial x}-f(\xi, x)\right] d \xi
\end{aligned}
$$

iterasyon formülü elde edilir.

$u(t, x)$ çözümünün ardışık yaklaşımları olan $u_{n}(t, x), \mathrm{n}>0$ için elde edilen Lagrange çarpanı iterasyon formülü ve başlangıç tahmini $u_{0}(t, x)$ kullanılarak hızlı bir şekilde elde edilebilir. Böylece çözüm

$u(t, x)=\lim _{n \rightarrow \infty} u_{n}(t, x)$

şeklinde olur.

\section{Varyasyonel iterasyon metodu ile nümerik çözümler}

$\mathrm{Bu}$ kısımda varyasyonel iterasyon metodunu yukarıda belirttiğimiz prosedürlere uygun şekilde aşağıdaki örnek probleme uygulayacağız. Başlangıç değer koşulları aşağıdaki gibi verilen kesirli mertebeden telegraf kısmi diferansiyel denklemin

$$
\left\{\begin{array}{l}
\frac{\partial^{2} u(t x)}{\partial t^{3}}+\frac{\partial^{\alpha} u(t, x)}{\partial t^{\alpha}}+u(t, x)=\frac{\partial^{2} u(t x)}{\partial x^{3}}+\frac{\partial u(t x)}{\partial x}+f(t, x), 0<x<\pi, 0<t<T, \\
f(t, x)=\left(2+6 t+2 t^{3}+\frac{6 t^{3-\alpha}}{\Gamma(4-\alpha)}\right) \sin x-\left(t^{3}+1\right) \cos x, \\
u(0, x)=\sin x, u_{t}(0, x)=0,0 \leq x \leq \pi, \\
u(t, 0)=u(t, L)=0,0 \leq t \leq T
\end{array}\right.
$$

nümerik çözümlerini bulmak için varyasyonel iterasyon metodunu uygulayalım. Verilen denklem için doğrulama fonksiyoneli genel hatlarıyla

$$
\begin{aligned}
u_{n+1}(t, x)= & u_{n}(t, x) \\
& +\int_{0}^{t} \lambda(\xi)\left[\frac{\partial^{2} u_{n}(\xi, x)}{\partial \xi^{2}}+\frac{\partial^{\alpha} u_{n}(\xi, x)}{\partial \xi^{\varpi}}+u_{n}(\xi, x)-\frac{\partial^{2} u_{n}(\xi, x)}{\partial x^{2}}\right. \\
& \left.-\frac{\partial u_{n}(\xi, x)}{\partial x}-f(\xi, x)\right] d \xi
\end{aligned}
$$

formülü ile ifade edilir. (2.7) denkleminde Lagrange çarpanının değeri

$\lambda(\xi)=\xi-t$ 
olarak bulunmuştu. Sonuç olarak iterasyon formülü

$$
\begin{aligned}
u_{n+1}(t, x)= & u_{n}(t, x) \\
& +\int_{0}^{t}(\xi-t)\left[\frac{\partial^{2} u_{n}(\xi, x)}{\partial \xi^{2}}+\frac{\partial^{\alpha} u_{n}(\xi, x)}{\partial \xi^{\alpha}}+u_{n}(\xi, x)-\frac{\partial^{2} u_{n}(\xi, x)}{\partial x^{2}}\right. \\
& \left.-\frac{\partial u_{n}(\xi, x)}{\partial x}-f(\xi, x)\right] d \xi
\end{aligned}
$$

şeklinde yazılır. Varyasyonel iterasyon metodunun prosedürlerine uygun olarak (2.2) ifadesinde belirtilen başlangıç tahmininden ve (3.1) ifadesindeki başlangıç değer koşullarından

$u_{0}(t, x)=\varphi_{1}(t, x)+t \varphi_{2}(t, x)$

olup buradan da

$u_{0}(t, x)=\sin x$

şeklinde bulunur. Bu son denklem de

$u_{1}(t, x)=u_{0}(t, x)+\int_{0}^{t}(\xi-t)\left[\frac{\partial^{2} u_{0}(\xi, x)}{\partial \xi^{2}}+\frac{\partial^{\alpha} u_{0}(\xi, x)}{\partial \xi^{\alpha}}+u_{0}(\xi, x)-\frac{\partial^{x_{u_{0}}(\xi, x)}}{\partial x^{2}}-\frac{\partial u_{0}(\xi, x)}{\partial x}-\right.$ $f(\xi, x)] d \xi$

olarak yazılabilir. Şimdi (3.1) daki başlangıç değer koşulları ve (3.4) deki $u_{0}(t, x)$ değeri ve türevleri bu son denklemde yerine yazılırsa

$$
\begin{aligned}
& u_{1}(t, x)=\sin x+\int_{0}^{t}(\xi-t)\left[\sin x+\sin x-\cos x-\left(2+6 \xi+2 \xi^{3}+\frac{6 \xi^{3-\alpha}}{\Gamma(4-\alpha)}\right) \sin x+\right. \\
& \left.\left(\xi^{3}+1\right) \cos x\right] d \xi
\end{aligned}
$$

bulunur. Sadeleştirmeleri yapıp $(\xi-t)$ ifadesini iç kısma dağıtılır,

$$
\begin{aligned}
& u_{1}(t, x)=\sin x+\int_{0}^{t}\left[\left(-6 \xi^{2}-2 \xi^{4}-\frac{6 \xi^{4-\alpha}}{\Gamma(4-\alpha)}+6 t \xi+2 t \xi^{3}+\frac{6 t \xi^{a-\alpha}}{\Gamma(4-\alpha)}\right) \sin x+\right. \\
& \left.\left(\xi^{4}-t \xi^{3}\right) \cos x\right] d \xi
\end{aligned}
$$

son ifadenin integrali alınır ve gerekli düzenlemeler yapılırsa

$$
\begin{aligned}
& u_{1}(t, x)=\sin x+\left[\left(-2 t^{3}-\frac{2 t^{5}}{5}-\frac{6 t^{5-\alpha}}{\Gamma(4-\alpha)(5-\alpha)}+3 t^{3}+\frac{t^{5}}{2}+\frac{6 t^{5-\alpha}}{\Gamma(5-\alpha)}\right) \sin x+\left(\frac{t^{5}}{5}-\right.\right. \\
& \left.\left.\frac{t^{5}}{4}\right) \cos x\right]
\end{aligned}
$$

olur. Buradan da gerekli sadeleştirmeler yapılırsa

$$
u_{1}(t, x)=\left[\left(1+t^{3}+\frac{t^{5}}{10}+\frac{6 t^{5-\alpha}}{\Gamma(6-\alpha)}\right) \sin x-\frac{t^{5}}{20} \cos x\right]
$$


şeklinde elde edilir. Elde edilen bu tespitle ve aynı hareket tarzıyla ardışık yaklaşımları bulmaya devam edelim. (3.3) denkleminde $n=1$ olarak alınırsa

$u_{2}(t, x)=u_{1}(t, x)+\int_{0}^{t}(\xi-t)\left[\frac{\partial^{\mathbb{2}} u_{1}(\xi, x)}{\partial \xi^{2}}+\frac{\partial^{\alpha} u_{1}(\xi, x)}{\partial \xi^{\alpha}}+u_{1}(\xi, x)-\frac{\partial^{2} u_{1}(\xi, x)}{\partial x^{2}}-\frac{\partial u_{1}(\xi, x)}{\partial x}-\right.$ $f(\xi, x)] d \xi$

olur. (3.1) denkleminde başlangıç değer koşulları ve (3.5) denkleminde $u_{1}(t, x)$ değeri ve türevleri bu son denklemde yerine yazılırsa

$$
\begin{aligned}
u_{2}(t, x)=[( & \left.\left.+t^{3}+\frac{t^{5}}{10}+\frac{6 t^{5-\alpha}}{\Gamma(6-\alpha)}\right) \sin x-\frac{t^{5}}{20} \cos x\right] \\
& +\int_{0}^{t}(\xi-t)\left[\left(6 \xi+2 \xi^{3}+\frac{6 \xi^{3-\alpha}}{\Gamma(4-\alpha)}\right) \sin x-\xi^{3} \cos x\right. \\
& +\left(\frac{6 \xi^{3-\alpha}}{\Gamma(4-\alpha)}+\frac{12 \xi^{5-\alpha}}{\Gamma(6-\alpha)}+\frac{6 \Gamma(6-\alpha) \xi^{5-2 \alpha}}{\Gamma(6-\alpha) \Gamma(6-2 \alpha)}\right) \sin x \\
& -\frac{6 \xi^{5-\alpha}}{\Gamma(6-\alpha)} \cos x+\left(1+\xi^{3}+\frac{\xi^{5}}{10}+\frac{6 \xi^{5-\alpha}}{\Gamma(6-\alpha)}\right) \sin x-\frac{\xi^{5}}{20} \cos x \\
& +\left(1+\xi^{3}+\frac{\xi^{5}}{10}+\frac{6 \xi^{5-\alpha}}{\Gamma(6-\alpha)}\right) \sin x-\frac{\xi^{5}}{20} \cos x \\
& -\left(1+\xi^{3}+\frac{\xi^{5}}{10}+\frac{6 \xi^{5-\alpha}}{\Gamma(6-\alpha)}\right) \cos x-\frac{\xi^{5}}{20} \sin x \\
& \left.-\left(2+6 \xi+2 \xi^{3}+\frac{6 \xi^{3-\alpha}}{\Gamma(4-\alpha)}\right) \sin x+\left(1+\xi^{3}\right) \cos x\right] d \xi
\end{aligned}
$$

ve gerekli işlemler yapılırsa

$$
u_{2}(t, x)=\left(1+t^{3}-\frac{t^{7}}{280}-\frac{24 t^{7-\alpha}}{\Gamma(8-\alpha)}-\frac{6 t^{7-\pi \alpha}}{\Gamma(8-2 \alpha)}\right) \sin x+\left(\frac{t^{7}}{210}+\frac{12 t^{7-\alpha}}{\Gamma(8-\alpha)}\right) \cos x
$$

elde edilir. Şimdi (3.3) denkleminde $n=2$ olarak alıp aynı işlemleri uygularsak

$$
\begin{aligned}
& u_{3}(t, x)=\left(1+t^{3}-\frac{t^{7}}{280}-\frac{24 t^{7-\alpha}}{\Gamma(8-\alpha)}-\frac{6 t^{7-\alpha \alpha}}{\Gamma(8-2 \alpha)}\right) \sin x+\left(\frac{t^{7}}{210}+\frac{12 t^{7-\alpha}}{\Gamma(8-\alpha)}\right) \cos x+ \\
& \int_{0}^{t}(\xi-t)\left[\left(6 \xi-\frac{3 \xi^{5}}{20}-\frac{24 \xi^{5-\alpha}}{\Gamma(6-\alpha)}-\frac{6 \xi^{5-\alpha \alpha}}{\Gamma(6-2 \alpha)}\right) \sin x+\left(\frac{\xi^{5}}{5}+\frac{12 \xi^{5-\alpha}}{\Gamma(6-\alpha)}\right) \cos x+\left(\frac{6 \xi^{3-\alpha}}{\Gamma(4-\alpha)}-\right.\right. \\
& \left.\frac{18 \xi^{7-\alpha}}{\Gamma(8-\alpha)}-\frac{24 \Gamma(8-\alpha) \xi^{7-2 \alpha}}{\Gamma(8-\alpha) \Gamma(8-2 \alpha)}-\frac{6 \Gamma(8-2 \alpha) \xi^{7-a \alpha}}{\Gamma(8-2 \alpha) \Gamma(8-3 \alpha)}\right) \sin x+\left(\frac{24 \xi^{5-\alpha}}{\Gamma(6-\alpha)}+\frac{12 \Gamma(6-\alpha) \xi^{5-2 \alpha}}{\Gamma(6-\alpha) \Gamma(6-2 \alpha)}\right) \cos x+ \\
& \left(1+\xi^{3}-\frac{\xi^{7}}{280}-\frac{24 \xi^{7-\alpha}}{\Gamma(8-\alpha)}-\frac{6 \xi^{7-2 \alpha}}{\Gamma(8-2 \alpha)}\right) \sin x+\left(\frac{\xi^{7}}{210}+\frac{12 \xi^{7-\alpha}}{\Gamma(8-\alpha)}\right) \cos x+\left(1+\xi^{3}-\frac{\xi^{7}}{280}-\right. \\
& \left.\frac{24 \xi^{7-\alpha}}{\Gamma(8-\alpha)}-\frac{6 \xi^{7-a \alpha}}{\Gamma(8-2 \alpha)}\right) \sin x+\left(\frac{\xi^{7}}{210}+\frac{12 \xi^{7-\alpha}}{\Gamma(8-\alpha)}\right) \cos x-\left(1+\xi^{3}-\frac{\xi^{7}}{280}-\frac{24 \xi^{7-\alpha}}{\Gamma(8-\alpha)}-\right. \\
& \left.\frac{6 \xi^{7-2 \alpha}}{\Gamma(8-2 \alpha)}\right) \cos x+\left(\frac{\xi^{7}}{210}+\frac{12 \xi^{7-\alpha}}{\Gamma(8-\alpha)}\right) \sin x-\left(2+6 \xi+2 \xi^{3}+\frac{6 \xi^{3-\alpha}}{\Gamma(4-\alpha)}\right) \sin x+ \\
& \left.\left(1+\xi^{3}\right) \cos x\right] d \xi
\end{aligned}
$$

bulunur. Buradan da gerekli işlemler yapılırsa 
$\mathrm{u}_{3}(\mathrm{t}, \mathrm{x})=\left(1+\mathrm{t}^{3}+\frac{\mathrm{t}^{9}}{30240}+\frac{54 \mathrm{t}^{9-\alpha}}{\Gamma(10-\alpha)}+\frac{36 \mathrm{t}^{9-2 \alpha}}{\Gamma(10-2 \alpha)}+\frac{6 \mathrm{t}^{9-3 \alpha}}{\Gamma(10-3 \alpha)}\right) \sin \mathrm{x}-\left(\frac{11 \mathrm{t}^{9}}{60480}+\frac{24 \mathrm{t}^{7-\alpha}}{\Gamma(8-\alpha)}+\right.$
$\left.\frac{12 \mathrm{t}^{7-2 \alpha}}{\Gamma(8-2 \alpha)}+\frac{48 \mathrm{t}^{9-\alpha}}{\Gamma(10-\alpha)}+\frac{6 \mathrm{t}^{9-2 \alpha}}{\Gamma(10-2 \alpha)}\right) \cos \mathrm{x}$

sonucu bulunur. İşlem zorluğundan dolayı sonraki adımlar elde etmek oldukça güçtür. Laplace dönüşüm metodu kullanılarak (3.1) probleminin tam çözümünün $u(t, x)=\left(t^{3}+1\right) \sin x$

olduğu kolaylıkla görülebilir. Matlab programını kullanarak tam ve yaklaşık çözümler için aşağıdaki grafikler elde edilir.

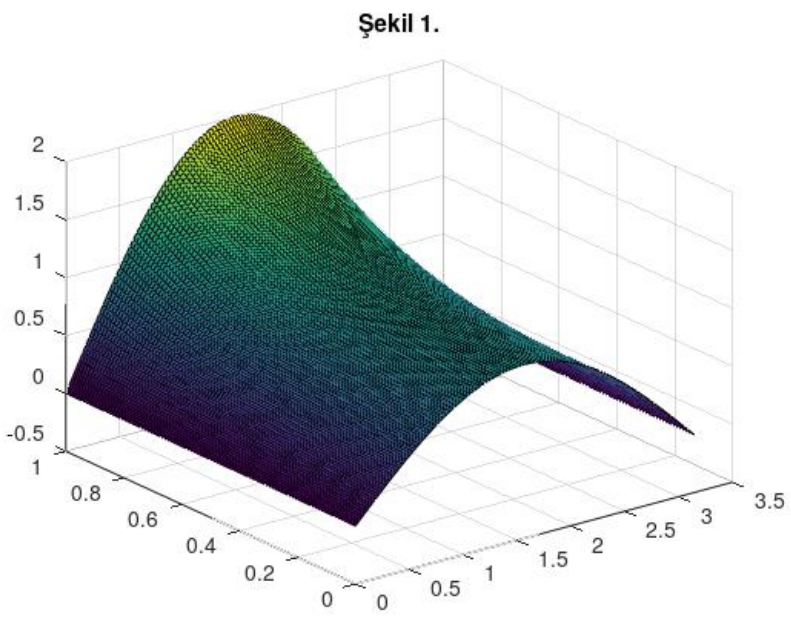

Şekil $1.0 \leq x \leq \pi$ ve $0 \leq t \leq 1$ aralığında tam çözümün grafiğini verir.

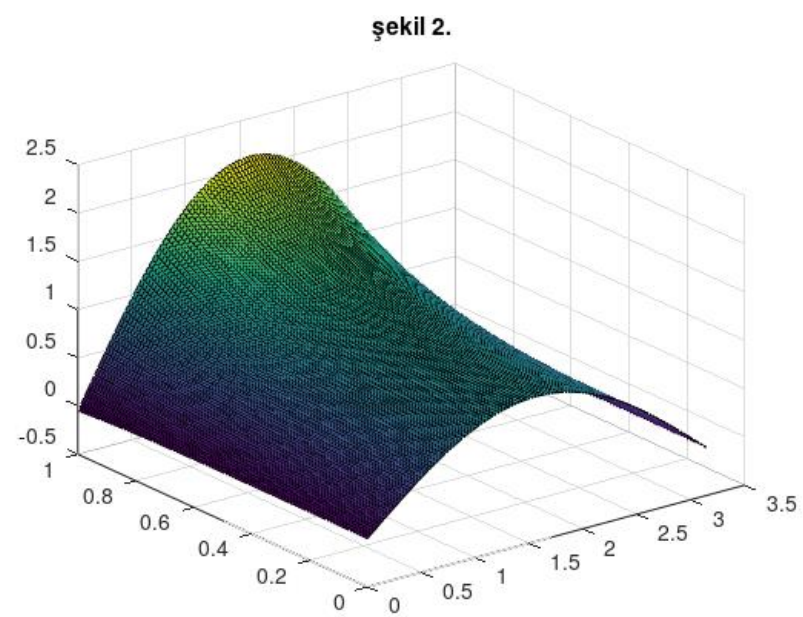

Şekil 2. Varyasyonel iterasyon metodu ile $n=1, \alpha=0.1$ ve $0 \leq x \leq \pi, 0 \leq t \leq 1$ aralığındaki grafiğini verir. 


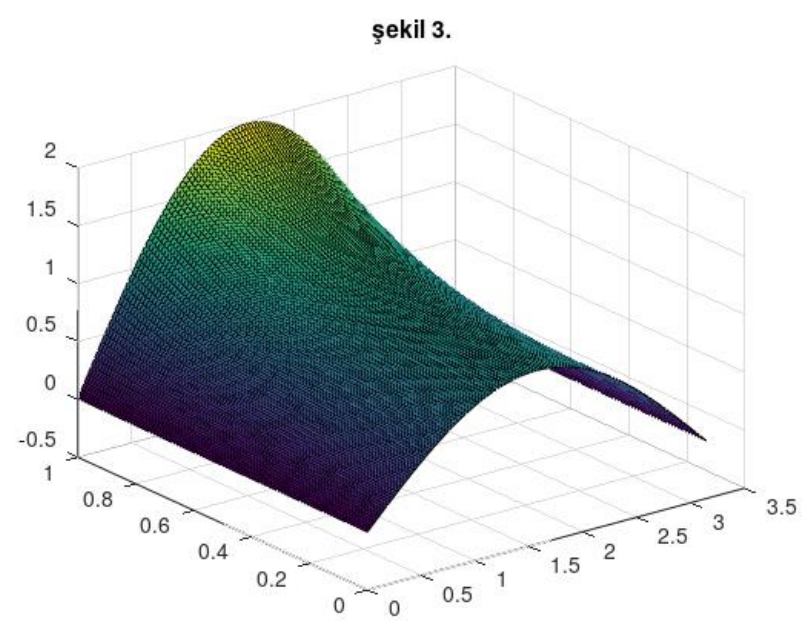

Şekil 3. Varyasyonel iterasyon metodu ile $n=2, \alpha=0.1$ ve $0 \leq x \leq \pi, 0 \leq t \leq 1$ aralığgındaki grafiğini verir.

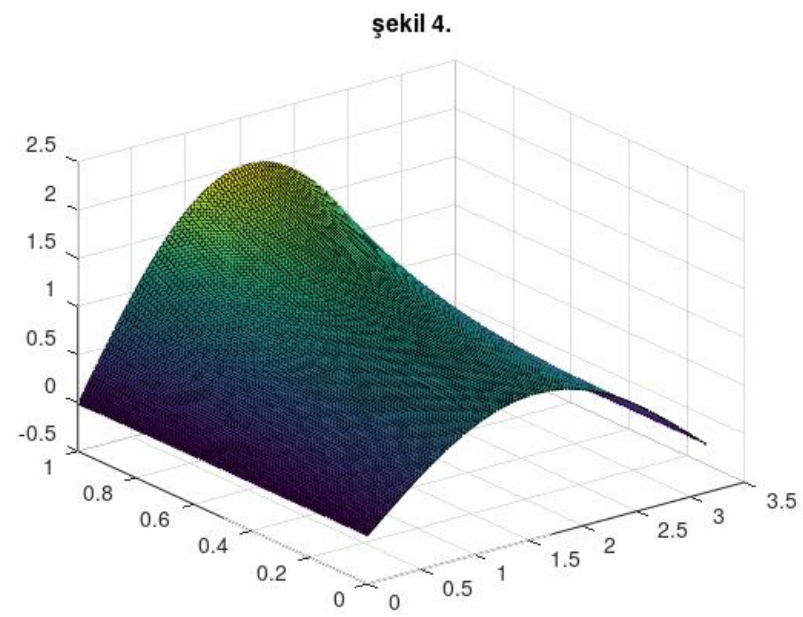

Şekil 4. Varyasyonel iterasyon metodu ile $n=3, \alpha=0.1$ ve $0 \leq x \leq \pi, 0 \leq t \leq 1$ aralığındaki grafiğini verir.

\section{Sonuç}

$\mathrm{Bu}$ çalışmada kesirli mertebeden telegraf kısmi diferansiyel denklemin varyasyonel iterasyon metoduyla çözümü ele alındı. Bu metot kullanılarak, her türden lineer ya da lineer olmayan kesirli mertebede kısmi diferansiyel denklemlerin ve yüksek mertebeden kısmi diferansiyel denklemlerin ardışık yaklaşımlarına adım adım ulaşılabilir ve nümerik çözümleri elde edilebilir. Metot, analitik çözümü olmayan problemlerde ve non-lineerliği kuvvetli olan problemlerde sayıca fazla iterasyonla ve zorlayıc1 çözümlerle olsa da bir dizi iterasyon sonucu çözüme hassas bir şekilde yakınsayabildiği görülmektedir. Çözümlerin yakınsama hassaslığı, başlangıç yaklaşımının ve Lagrange çarpanının iyi tayin edilmesiyle ilgilidir. Bu metodun kesirli mertebeden telegraf kısmi denklemine uygulanması bu çalışmayı farklı yapmaktadır. Şekiller karşılaştırıldığında, Şekil 1. tam çözümün grafiği ile Şekil 2., Şekil 3. ve Şekil 4. yaklaşık çözümlerin her adım için hesaplanan grafiklerinin birbirine oldukça yakın olduğu görülür. Böylece 
verilen metodun bu kesirli mertebeden telegraf kısmi diferansiyel denklemin yaklaşık çözümü için elverişli ve iyi sonuçlar verdiği söylenebilir.

\section{Kaynaklar}

[1] Podlubny, I., Fractional Differential Equations, vol. 198 of Mathematics in Science and Engineering, Academic Press, San Diego, Calif, USA, (1999).

[2] Samko, S.G., Kilbass A.A., ve Marchiev, O.I., Fractional integrals and derivatives, Theory and Applications, Gordon and Breach, Yverdon, 973s., (1993).

[3] Kılbass, A., Srivastava, H., ve Truj1llo, J., Theory and applications of fractional differential equations, North-Holland Mathematics Studies 204, NorthHolland, 521 pp, (2006).

[4] Caputo, M., ve Mainardi, F., Linear models of dissipation in anelastic solids, La Rivista del Nuovo Cimento, 1, 2, 161-198, (1971).

[5] He, J.-H., Nonlinear oscillation with fractional derivative and its applications, in Proceedings of the International Conference on Vibrating Engineering 98, vol. 9, Dalian, China, (1998).

[6] He, J.-H., Homotopy perturbation technique, Computer Methods in Applied Mechanics and Engineering, 178, 3-4, 257-262, (1999).

[7] He, J.-H., Approximate analytical solution for seepage flow with fractional derivatives in porous media, Computer Methods in Applied Mechanics and Engineering, 167, 1-2, 57-68, (1998).

[8] Barone, A., Esposito, F., Magee, C. J., ve Scott, A. C., Theory and applications of the sine-gordon equation, La Rivista del Nuovo Cimento, 1(2): 227-267, (1971).

[9] Yusufoglu, E., The variational iteration method for studying the Klein- Gordon equation, Applied Mathematics Letters, 21, 7, 669-674, (2008).

[10] Khan, M., Hyder Ali, S., ve Qi, H., On accelerated flows of a viscoelastic fluid with the fractional Burgers' model, Nonlinear Analysis. Real World Applications. An International Multidisciplinary Journal, 10, 4, 2286-2296, (2009).

[11] Elbeleze, A. A., K1lıçman A., ve Taib, B. M., Homotopy perturbation method for fractional black-scholes european option pricing equations using Sumudu transform, Mathematical Problems in Engineering, vol. 2013, Article ID 524852, 7 pages, (2013).

[12] Wu, J., Theory and Applications of Partial Functional Differential Equations, Springer, New York, NY, USA, (1996).

[13] Keller, A. A., Contribution of the delay differential equations to the complex economic macrodynamics, WSEAS Transactions on Systems, 9, 4, 358-371, (2010).

[14] Sakar, M. G., Uludag, F., ve Erdogan, F., Numerical solution of time-fractional nonlinear PDEs with proportional delays by homotopy perturbation method, Applied Mathematical Modelling, 40, 13-14, 6639-6649, (2016).

[15] Liu, J., ve Hou, G., Numerical solutions of the space- and time-fractional coupled Burgers equations by generalized differential transform method, Applied Mathematics and Computation, 217, 16, 7001-7008, (2011). 
[16] Momanı, S., ve Odibat, Z., Analytical solution of a time-fractional NavierStokes equation by Adomian decomposition method, Applied Mathematics and Computation, 177, 2, 488-494, (2006).

[17] Sakar, M. G., ve Erdogan, F., The homotopy analysis method for solving the time-fractional Fornberg-Whitham equation and comparison with Adomian's decomposition method, Applied Mathematical Modelling, 37, 20-21, 88768885, (2013).

[18] Kumar, S., ve Kumar, D., Fractional modelling for BBM-Burger equation by using new homotopy analysis transform method, Journal of the Association of Arab Universities for Basic and Applied Sciences, 16, 16-20, (2014).

[19] Zubik-Kowal, B., Chebyshev pseudospectral method and waveform relaxation for differential and differential-functional parabolic equations. Applied Numerical Mathematics, 34, 2-3, 309-328, (2000).

[20] Kumar, S., Kumar, D., Abbasbandy, S., ve Rashıd1, M. M., Analytical solution of fractional Navier-Stokes equation by using modified Laplace decomposition method, Ain Shams Engineering Journal, 5, 2, 569-574, (2014).

[21] Kumar, S., Yıldırım, A., Khan, Y., Jafarı, H., Sayevand, K., ve We1, L., Analytical solution of fractional Black-Scholes European option pricing equation by using Laplace transform, Journal of Fractional Calculus and Applications, 2, 8, 1-9, (2012).

[22] Tanthanuch, J., Symmetry analysis of the nonhomogeneous inviscid Burgers equation with delay, Communications in Nonlinear Science and Numerical Simulation, 17, 12, 4978-4987, (2012).

[23] Kurulay, M., The approximate and exact solutions of the space and timefractional Burggres equations, International Journal of Research and Reviews in Applied Sciences, 3, 3, 257-263, (2010).

[24] Momani, S., Erjaee, G. H., ve Alnasr, M. H., The modified homotopy perturbation method for solving strongly nonlinear oscillators, Computers and Mathematics with Applications, 58, 11-12, 2209-2220, (2009).

[25] Singh, J., Kumar, D., ve Kilıçman, A., Homotopy perturbation method for fractional gas dynamics equation using Sumudu transform, Abstract and Applied Analysis, vol. 2013, Article ID 934060, 8 pages, (2013).

[26] Golmankhaneh, A. K., Golmankhaneh, A. K., ve Baleanu, D., On nonlinear fractional KleinGordon equation, Signal Processing, 91, 3, 446-451, (2011).

[27] Kurulay, M., Solving the fractional nonlinear Klein-Gordon equation by means of the homotopy analysis method, Advances in Difference Equations, p. 2012, (2012).

[28] Yang, X. J., Baleanu, D., Khan, Y., ve Mohyud-Din, S. T., Local fractional variational iteration method for diffusion and wave equations on Cantor sets, Romanian Journal of Physics, 59, 1-2, 36-48, (2014).

[29] Geng, F., Lin, Y., ve Cu1, M., A piecewise variational iteration method for Riccati differential equations, Computers and Mathematics with Applications, 58, 11-12, 2518-2522, (2009).

[30] Prakash, A., Kumar, M., ve Sharma, K. K., Numerical method for solving fractional coupled Burgers equations, Applied Mathematics and Computation, 260, 314-320, (2015).

[31] Sakar, M. G., ve Ergören, H., Alternative variational iteration method for solving the time-fractional Fornberg-Whitham equation, Applied Mathematical Modelling, 39, 14, 3972-3979, (2015). 
[32] Singh, B. K., ve Kumar, P., Fractional Variational Iteration Method for Solving Fractional Partial Differential Equations with Proportional Delay, International Journal of Differential Equations, vol. 2017, Article ID 5206380, 11 pages, (2017).

[33] Elbeleze, A. A., Kiliçman, A., ve Taib, B. M., Fractional Variational Iteration Method and Its Application to Fractional Partial Differential Equation, Mathematical Problems in Engineering, vol. 2013, Article ID 543848, 10 pages, (2013).

[34] Modanli, M., Faraj, B. M., ve Ahmed, F. W., Using matrix stability for variable telegraph partial differential equation, An International Journal of Optimization and Control: Theories \& Applications (IJOCTA), 10(2), 237243, (2020).

[35] Modanli, M. (2018). Kesirli telegraf kısmi diferansiyel denklemlerin fark şeması metodu ile nümerik çözümü, Balıkesir Üniversitesi Fen Bilimleri Enstitüsü Dergisi, 20(1), 440-449, (2018).

[36] Modanli, M., On the numerical solution for third order fractional partial differential equation by difference scheme method, An International Journal of Optimization and Control: Theories \& Applications (IJOCTA), 9(3), 1-5, (2019).

[37] Yavuz, M., ve Abdeljawad, T., Nonlinear regularized long-wave models with a new integral transformation applied to the fractional derivative with power and Mittag-Leffler kernel, Advances in Difference Equations, 2020(1), 1-18, (2020).

[38] Yavuz, M., ve Özdemir, N., Analysis of an epidemic spreading model with exponential decay law, Mathematical Sciences and Applications ENotes, 8(1), 142-154, (2020).

[39] Evirgen, F., Analyze the optimal solutions of optimization problems by means of fractional gradient based system using VIM, An International Journal of Optimization and Control: Theories \& Applications (IJOCTA), 6(2), 75-83, (2016).

[40] Sarp, Ü., Evirgen, F., ve İkikardeş, S., Applications of differential transformation method to solve systems of ordinary and partial differential equations, Balıkesir Üniversitesi Fen Bilimleri Enstitüsü Dergisi, 20(2), 135156, (2018).

[41] Yavuz, M., ve Özdemir, N., Comparing the new fractional derivative operators involving exponential and Mittag-Leffler kernel, Discrete \& Continuous Dynamical Systems-S, 13(3), 995, (2020).

[42] Yokus, A., Durur, H., Ahmad, H., Thounthong, P., ve Zhang, Y. F. Construction of exact traveling wave solutions of the Bogoyavlenskii equation by $\left(\mathrm{G}^{\prime} / \mathrm{G}, 1 / \mathrm{G}\right)$ expansion and $\left(1 / \mathrm{G}^{\prime}\right)$-expansion techniques, Results in Physics, 19, 103409, (2020).

[43] Yokus, A., Durur, H., ve Ahmad, H., Hyperbolic type solutions for the couple Boiti-Leon-Pempinelli system, Facta Universitatis, Series: Mathematics and Informatics, 35(2), 523-531, (2020).

[44] Yokus, A., Durur, H., Kaya, D., Ahmad, H., ve Nofal, T. A., Numerical Comparison of Caputo and Conformable Derivatives of Time Fractional Burgers-Fisher Equation. Results in Physics, 104247, (2021).

[45] Ahmad, H., Khan, T. A., Durur, H., Ismail, G. M., ve Yokus, A., Analytic approximate solutions of diffusion equations arising in oil pollution, Journal of Ocean Engineering and Science, 6(1), 62-69, (2021). 
[46] Koksal, M. E., Time and frequency responses of non-integer order RLC circuits, AIMS Mathematics, 4(1), 64-78, (2019).

[47] Koksal, M. E., Senol, M., ve Unver, A. K., Numerical simulation of power transmission lines, Chinese Journal of Physics, 59, 507-524, (2019).

[48] Modanli, M., Abdulazeez, S. T., ve Husien, A. M., On variational iteration method for solving pseudo-hyperbolic partial differential equations with nonlocal conditions, (under review). 